\title{
Influence of Spiritualistic Belief on Musical Performance in Traditional Ritual: Mahesak City Guardian Ceremony in Champasak
}

\author{
Chalermpol Ataso ${ }^{1} \&$ Pratompong Na Champasakdi ${ }^{1}$ \\ ${ }^{1}$ Faculty of Cultural Science, Mahasarakam University, Thailand \\ Correspondence: Chalermpol Ataso, Faculty of Cultural Science, Mahasarakham University, Talard Sub-District, \\ Mueang Maha Sarakham District, 44000, Maha Sarakham Province, Thailand. Tel: 6688-321-9654. E-mail: \\ chalermpol.ata@gmail.com
}

Received: October 3, 2018

Accepted: October 26, 2018

Online Published: December 31, 2018

doi:10.5539/ach.v11n1p23

URL: http://dx.doi.org/10.5539/ach.v11n1p23

\begin{abstract}
In early March, Mahesak city guardian ceremony is yearly held in Champasak, Lao People's Democratic Republic to commemorate the city guardian spirits that were previously believed to protect Lao ancestors from unexplainable natural phenomena. Since the melody of music was believed to mediate the communication between human-beings and invisible city guardians, the sound of Piphat ensemble has become the heart of Mahesak ceremony. Therefore, in this study, the details of significance and religious implication of Mahesak ceremonial music via the sound of Piphat were elucidated. The variety in tunes and rhythms of Mahesak ceremonial songs exhibit the hierarchy of sacred sensations to worship different city guardians with the use of spiritual mediators as sequential representatives of each city guardian. These matters reflect the combination of primordial belief in guardian spirits amongst Lao people, despite the predominance of Buddhism in Lao People's Democratic Republic nowadays.
\end{abstract}

Keywords: Piphat, Mahesak, City Guardians, Champasak, Laos Music

\section{Introduction}

Ethnic Lao, formerly known as Tai ethnic group, is believed to originate in Southern China and relocate to Southeast Asia. After the invasion of the Chinese colonialism, the ethnic Lao ancestors were expelled and forced to migrate south to Muang Sawa (or Muang Soa), nowadays known as Luang Prabang (Church, 2006). In the old times, natural phenomena, such as rain, flood, thunder, drought, and plagues, brought fears to the ethnic Lao, since the nature was not yet well scientifically proved. The ancient Lao people therefore worshipped the city guardian spirits in order to relieve their fears of the unexplained phenomena. This had led to the formation of the ritual and ceremony performances that allowed Lao people to communicate with the city guardian spirits for pleading them happiness and protections (Thammawat, 1985). Phi Than, the sacred guardian existing in heaven, is the main city guardian whom has been venerated by Lao people for many centuries. The parietal arts in Dong Son cultural area of Northern Vietnam illiterate the use of musical performances for conveying their messages to the invisibles such as Phi Than, choir of angels, ancestral spirits, and city guardian spirits. Furthermore, the evidence from bronze-casted musical instruments indicates that the ceremonial music has been performed by ethnic Lao since prehistoric times (Heidhues, 2000). For instance, Kong-Bung, a 3,000 years old gong-shaped musical instrument made of bronze, was widely found in Southern China, Northern Vietnam, Laos, and North-Eastern Thailand (Heidhues, 2000).

In 14th century, Buddhism was firstly introduced to Laos from Intapatnakorn, nowadays known as Cambodia, by King Fa Ngum (Coedès, 1968). The ethnic Lao's original belief has been gradually influenced by Buddhism, causing the musical instruments and performances to evolve. Alongside the predominance of Buddhism, Piphat started to play a major role in ritual and ceremonial musical performances of Laos royalty (Wisuttipat, 2014). The ancient bronze-carving musical instruments had been slowly replaced by Piphat in both Luang Prabang and Vientiane royalties. Later, Prakru Ponsamek (Yakru-Kheehom) escorted 3,000 people from Vientiane to establish the new city called Champasak which has become the third city of Laos (Wipakpotjanakit, 2003).

In 1713, Champasak was founded by the reign of primary king Chaosoisri Samutpootthangkoon, the descendant of Prachaosuriyawongsathammikgarat (the king of Vientiane), and became the capital city of Southern Laos (Burke \& Vaisutis, 2007). The musical performances during the reign of Chaosoisri Samutpootthangkoon was 
evidenced from the royal credential informing the arrangement for the royal cremation of Chaoratchakruluang Yodkeaw Ponsamek (higher royal rank of former Yakru-Kheehom). The credential revealed the king's mandate to organize the musical performances to commemorate the late Yakru-Kheehom. In Champasak, Piphat ensemble was preserved under the regulation of the royal house Khum Phookrongnakorn to be performed in various rites and traditional ceremonies in accordance with either Buddhism and ethnic Lao's original belief. Despite the predominance of Buddhism, people in Southern Laos still appear to believe in city guardian spirits, since the music for worshipping and communicating with the invisibles, such as Mahesak city guardian ceremony, remains widely performed in Southeast Asia, especially in Southern Laos.

In the past, Mahesak city guardian ceremony, a long historic ritual performance of Champasak, raised the opportunity for Champasak royal family and Laos civilians to participate in the same event. Up to now, Champasak civilians have held Mahesak city guardian ceremony each year in order to show their respect towards the city guardian spirits, since they believe that this ceremony would allow them to communicate with the invisibles, Phi Than, through the sound of Piphat (Campbell, 1991). The performance of Piphat ensemble is carried out in the ceremony to summon the city guardian spirits to possess the male and female spiritual mediators, called Poe-Sang and Mae-Lam, respectively, to enable Champasak civilians to offer tributes and plead for prosperity and happiness (Hanna, 1988). Mahesak city guardian ceremony has become an intriguing sacred rite that expresses the faith of Champasak civilians in the city guardian spirits by using the sound of Piphat as a medium.

The performance of Piphat ensemble was previously patronized by the Champasak royalty. The movement of communist party led to a significant revolution of Laos in 1975, also known as socialist transformation, resulting in a long halt of Mahesak city guardian ceremony (Brown \& Zasloff, 1976). At present, Laos government has been promoting arts and music, and encouraging the restoration of Mahesak city guardian ceremony. The Piphat ensemble has been adjusted and modified conforming to the urbanization of Lao People's Democratic Republic (Pikulsri, 2008). This study mainly focused on the investigation of the details of Piphat ensemble and the ritual songs played during Mahesak city guardian ceremony. As a result, the conservation awareness of this valuable traditional music would be raised and the knowledge would be passed on to the new generation.

\section{Objectives}

(1) To investigate the current status of Piphat ensemble and the musical instruments used in Mahesak city guardian ceremony in Champasak.

(2) To study the roles of the ritual songs played during Mahesak city guardian ceremony.

\section{Methodology}

\subsection{Target Groups}

Throughout the investigation, multiple sessions of fieldwork were performed to collect the data from the prototypical sources in Champasak, Champasak Province, Lao People's Democratic Republic. In this study, the target groups were divided into two groups. The first target group was mainly focused on the interview sessions with ordinary Champasak civilians to obtain information about Mahesak city guardian ceremony. The interview sessions were intimately conducted with causal and informal manners to acquire the most insightful information about background, performance patterns and evolution of the ceremony from pre- to post-revolutionary period of Laos which reflects in the change in attitudes of Lao people towards Mahesak ceremony. For the second target group, the people directly involved in the ceremony were selected, including Poe-Sang, Mae-Lam, Khao Jam (subsidiary men of Poe-Sang), the musicians and the defectors, who were previously engaged with the ceremony. The private and grouped interviews were conducted to obtain in-depth information about the role of Piphat ensemble in the ceremony and evolution of Piphat ensemble from pre-revolutionary time until now.

\subsection{Data Collecting}

In this study, the results from the field study was validated by the data from Laos historical literature, academic textbooks and the relevant research articles. The sources of data including online database and university libraries were used in this investigation to proceed the fieldwork data analysis. The methods of data gathering were, as follows;

\subsubsection{Formal Interview Sessions}

The permission requests were, first, accepted by the target groups. During the sessions, the topics of conversation and questions were determined beforehand. The information obtained from the interview was rearranged and analyzed based on the study objectives. 


\subsubsection{Informal Interview Session}

The field study was conducted for multiple times to develop intimacy with Champasak villagers, since the large amount of information was rare and confidential. The conversations were performed in a causal manner to ask about the common issues such as life styles, work routine, and preferences in music. Later, the questions were gradually proceeded toward the specific matter about the music played in Mahesak city guardian ceremony.

\subsubsection{Observation with Participation}

The researcher volunteered to participate in arrangement of Mahesak city guardian ceremony by helping the villagers organize the ceremony area and assemble the Piphat ensemble.

\subsubsection{Observation without Participation}

The procedure of Mahesak city guardian ceremony was observed and recorded in the form of videos, pictures, and audios. The materials obtained from the field study were rearranged and analyzed according to the study objectives.

\subsection{Data Analysis}

The data of Mahesak city guardian ceremony, mainly obtained from the fieldwork, were categorized into two groups based on the study objectives shown as follows;

3.3.1 The investigation of the current status of Piphat ensemble and the musical instruments used in Mahesak city guardian ceremony in Champasak.

- The status of Piphat ensemble from the Kingdom of Laos era to the post-revolutionary times.

- The current status of the musician council.

- The present assembly of musical instruments in Mahesak city guardian ceremony.

3.3.2 The study of the role of songs played during Mahesak city guardian ceremony performance in Champasak.

- The elucidation of the songs played during Mahesak city guardian ceremony.

- The roles of songs in each part of Mahesak city guardian ceremony.

- The melodic patterns of the songs played for worshiping city guardian spirits.

The information obtained from the aforementioned objectives was analyzed and expressed in the form of descriptive writing including the supportive data from the relevant research articles and literatures.

\section{Results and Discussion}

In early March of each year, Mahesak city guardian ceremony is held by Champasak civilians in one day, from dusk till dawn, at 'Ho-Fon' pavilion located in the ceremonial area called Samosorn Banprapeen. Champasak people believe that the ceremony would enable them to venerate and communicate with the city guardian spirits upon their faith through male and female spiritual mediators, called Poe-Sang and Mae-larm (Figure 1), respectively (Bunpala, 2015). During the ceremony, the city guardian spirits, such as Inta lord, Kamsan-ngon lord, Thammarangsi lord, Paya Kammata, Poe-lan, and Great Naga, were offered with tributes and appealed to possess the spiritual mediators, allowing Champasak people to deliver their questions and requests to the city guardian spirits (Doungwilai et al., 2012). Every three years, the special Mahesak city guardian ceremony is held for a longer period of time, 2 days and 1 night, in order to prolong the opportunity for Champasak people to communicate with their venerated city guardian spirits. One of the two main musical instruments is Klong Lhuk, a barrel shaped-drum with leather stretched over the top and bottom surfaces (Figure 2). Mhong, another main musical instrument used in Mahesak ceremony, is a percussion instrument similar to Gong, but providing more vibrant sound (Figure 3). Apart from Piphat ensemble, both Klong Lhuk and Mhong are continually played for the entire ceremony from the beginning to the end to offer sacred sensations to the ceremony. Piphat ensemble performance is believed to play a crucial role in summoning the city guardian spirits to possess the spiritual mediators, Poe-Sang and Mae-Lam (Figure 4). After being possessed, the mediators will perform an unpatterned dance based on their pleasure to provide amusing and enjoyable feelings to the participating civilians (Hanna, 1988). Mahasak city guardian spirits ceremony is believed not only to bring prosperity and tranquility to Champasak but also to motivate Champasak civilians to live their lives cheerfully. Moreover, this Piphat ensemble was formerly used only in royalty of Chamapsak during the era of Kingdom of Laos. 


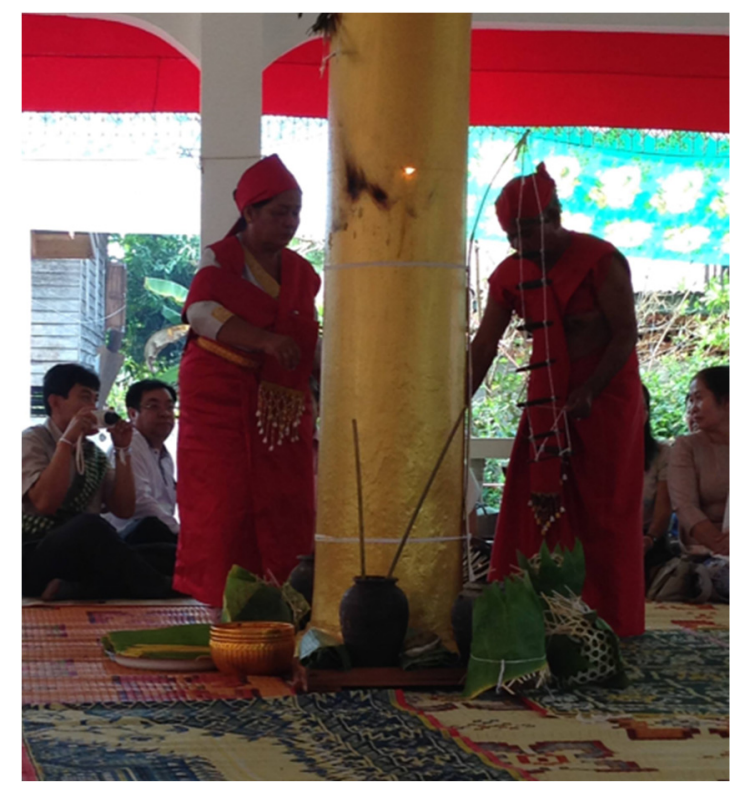

Figure 1. Poe-Sang and Mae-Lam

The spiritual mediators who conducted the communication between Mahesak city guardians and ceremonial participants.

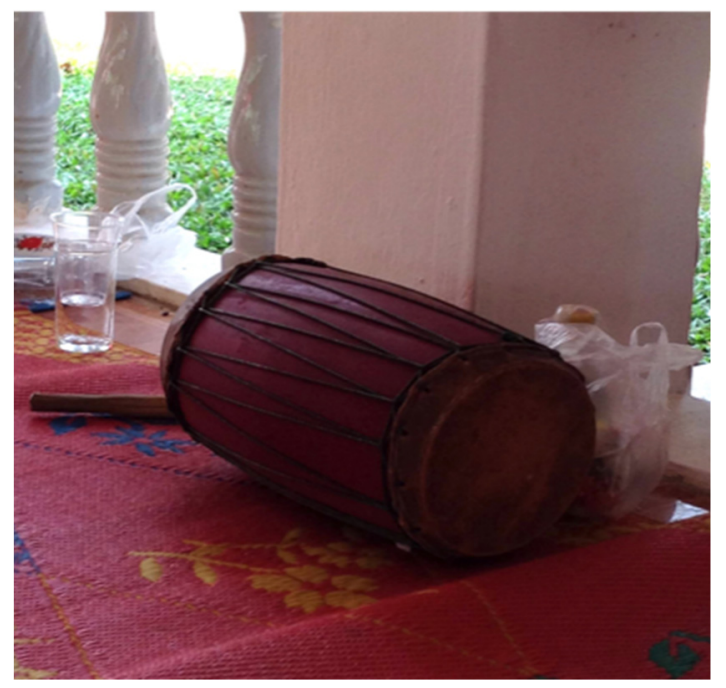

Figure 2. Klong Lhuk

The main percussion instrument that provides a sacred sensation to Mahesak city guardian ceremony. 


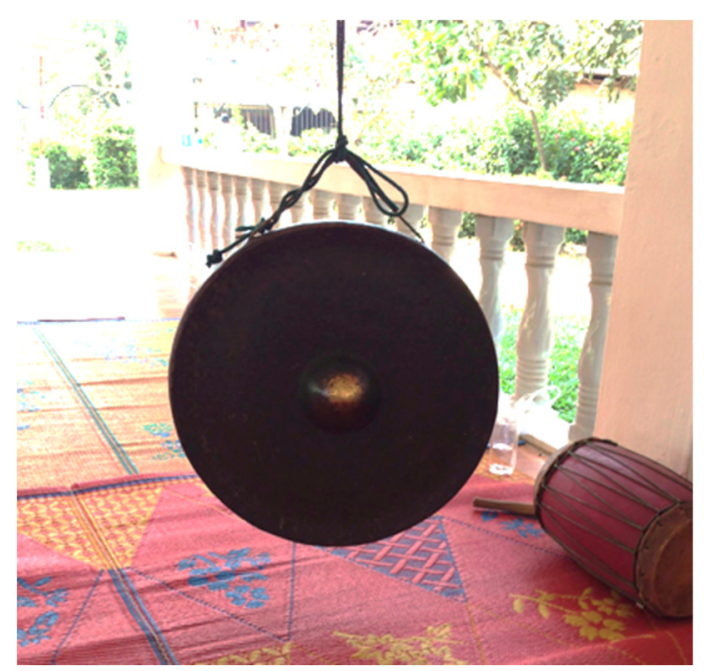

Figure 3. Mhong

The main percussion instrument that provides sacredly reverberating sound throughout Mahesak city guardian ceremony.

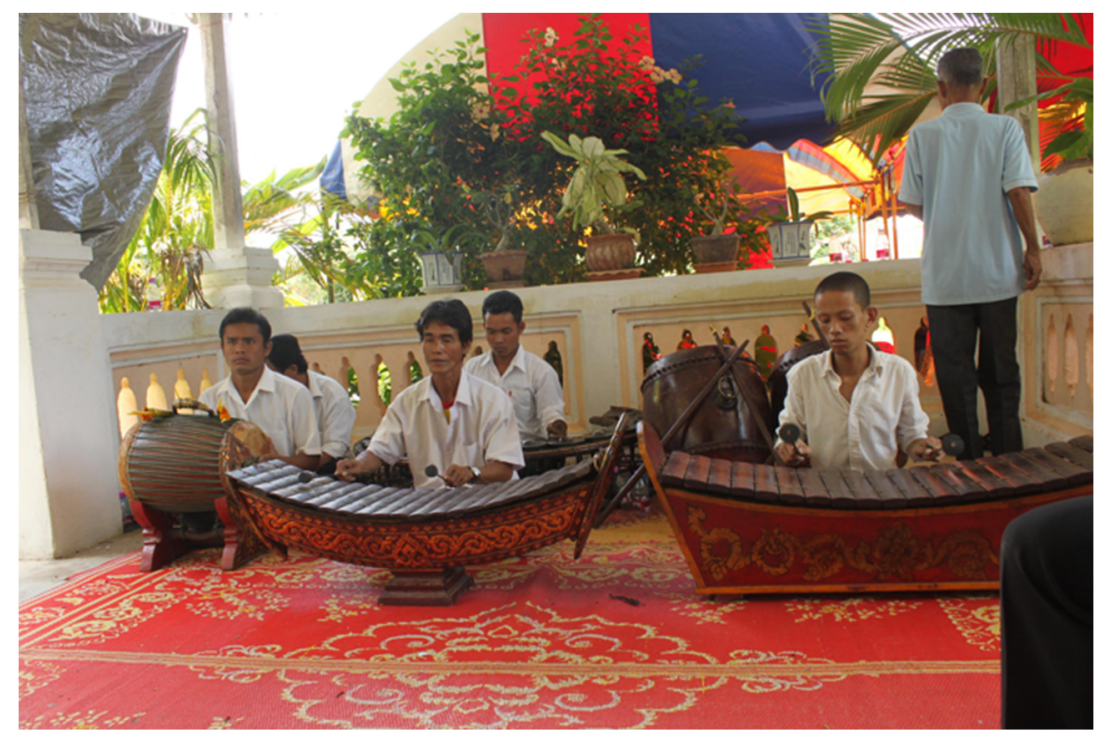

Figure 4. Piphat ensemble in Mahesak city guardian ceremony

After the revolution by communist party in 1975, the Laos royalty-related traditional practices were prohibited by the new government, thereby leading to the impediment of Piphat ensemble performance (Evans, 1998). During the revolutionary era, all the musical instruments of royal Piphat ensemble were detained by Laos government, whereas only some instruments were retrieved after the liberation of Laos people. Piphat ensemble was, once again, used for religious ceremonies and traditional rituals in Champasak. Nowadays, Piphat ensemble of Mahesak city guardian ceremony has been adjusted to suit with the civil modernization and social and political context transformation (Pikulsri, 2008). Eight musicians currently active in Piphat ensemble performance are Mr. Inpang Kaewponsai, Mr. Lodmhai Saitasit, Mr. Korakan Pommaleusai, Mr. Kaew Kambuppha, Mr. Salermsai Wongsin, Mr. Boonmi Norachak, Mr. Puangped Wongpachan, and Mr. Boonkerd Pommasing (the conductor of Piphat ensemble). The musical instruments, recently used in Mahesak city guardian ceremony, are comprised of several percussion and woodwind instruments shown as follows;

1) 'Ranat Ek' a wooden high-pitched xylophone derived from Thai Ranat Ek, since the original Laos Ranat Ek could not be retrieved from the government after the termination of revolutionary era. 
2) 'Ranat Thum' an original antique Laos wooden low-pitched xylophone carved with red and yellow carving lines, bolden with black lines, unveiling exquisite Laos-styled artistic patterns.

3) 'Kong Wong Yhai' a Thai-derived gong given by his Majesty's King Rama 5 of Thailand.

4) 'Ranat Lek' a metallophone embellished with Laos antique artistic patterns created by red and yellow colored-carving lines bolden with black lines.

5) 'Taphon' a two-headed barrel-shaped drum equipped with a wooden support and two handles on the upper side.

6) 'Klong Tat' a large barrel-shaped drum similar to Thai and Cambodian Klong Tat.

7) 'Pii”' a woodwind instrument similar to Thai Pii.

8) 'Ching' and 'Chab' small finger cymbals and large flat cymbals, respectively, which are similar to Thai and Cambodian versions.

All the listed musical instruments are stored at the music hall in Samosorn Banprapeen, the area where various religious ceremonies are performed. Many buildings and constructions are located in Samosorn Banprapeen area such as Ho-Fon pavilion, Mahesak hall, music hall, housings for Poe-Sang and Mae-Lam.

During Mahesak city guardian ceremony, the spiritual mediators, Poe-Sang and Mae-Lam, are sequentially dominated by different city guardian spirits at particular times, so the songs will be specifically played according to the requests of Poe-Sang and Mae-Lam. The sequence of song performance is commonly known among the musicians as it is run by the possession sequence of city guardian spirits that is conveyed through gestures of the spiritual mediators. In addition, there are a variety of songs particularly played for each distinct part of the ceremony such as Kud-lhao (booze drinking) and Yai-lhao (distribution of booze). Significance and implication from each step of the ceremony are expressed by the difference in melody and harmony of the songs played during the ceremony. During the ceremony, Klom song is played in an honor of Thammarangsi Lord, when possessing Poe-Sang, since it exhibits elegant melodies with 4 scales and consistent rhythms throughout the song (Figure 5).

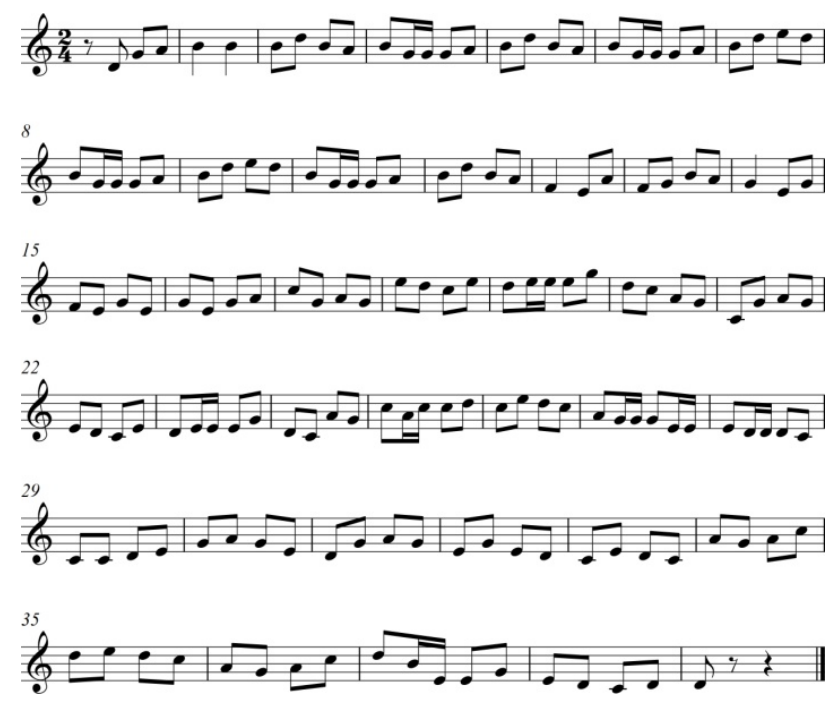

Figure 5. Melody of Klom Song Scale 1

Krawnok song also expresses the elegant feelings from its melodies with consistent rhythms which is played when Mae-Lam is possessed by Kamsanngon lord. Both of Klom and Krawnok songs are equipped with the sound of Taphon and Klong Tat by which the distinct and consistent rhythms are created. Orathai song serves as the song that provides pleasure and amusement for Mae-Lam when possessed by Kamsan-Ngon Lord. The cheerful and enjoyable melodies of Orathai song are played along with the dance of the possessed Mae-Lam. When the dance of Mae-Lam is prolonged, the musicians will attempt to maintain the amusement by playing other song called Nakkeaw song. Klom, Krawnok and Orathai songs are sequentially played in loops based on the presence of each city guardian spirits. 


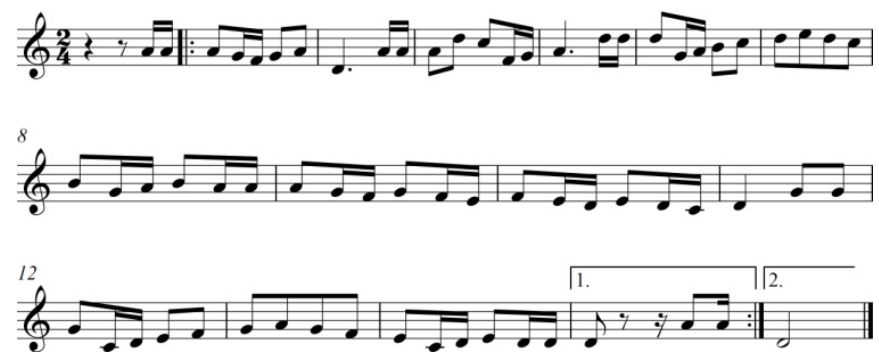

Figure 6. Melody of Orathai Song

The appearance of different city guardian spirits is anticipated by the musicians who also determine the songs to perform for conveying respectful messages to different city guardian spirits. The musicians will play Klom song immediately when Poe-Sang starts performing the dance, whereas Krawnok song will be played when Mae-Lam becomes active at her graceful dance. However, Orathai song will be played when Mae-Lam performs her dance with a sword in her hand. These three songs, therefore, play an essential role in the ceremony, since they are performed more frequently than the other songs. Apart from the main three songs, the other songs are also played for praising and venerating other city guardian spirits. The performance of Cherd song, consisting of exciting and invigorating melodies, is conducted when Poe-Sang's soul is occupied by Great Naga. The sweet and graceful tune of Nok Khan Si Nual song is played when the soul of Poe-Sang is embraced by Paya Kammata. These two songs are only played once during Mahesak ceremony, since Poe-Sang is occupied by Great Naga and Paya Kammata for only one time during the ceremony. Besides the aforementioned songs, performance of other ceremonial songs also signifies the matter of each step of Mahesak ceremony. Nangnak song, comprising the sweet-toned melodies, is played to express an auspicious aspect during the distribution of holy booze to ceremony participants. During this step, Mae-Lam carries a silver bowl, called Khan, containing holy booze and walk around to give the holy booze to Champasak civilians who attended the event. Kamen Keawkhao song, displaying simple, yet, elegant melodies, is performed during Kad Lhao part at which people who were previously related to the royal lineage of Champasak royalty will have a participation. In the morning session of Mahesak ceremony, the descendants of former Champasak royalty, who currently reside in Laos and other countries, normally participate in the Kad Lhao part by drinking the holy booze through straws from a jar located at the central pillar of Ho-Fon hall. Moreover, there are a number of songs found during Mahesak city guardian ceremony i.e. Kamen Saiyok song, Kamen Puang song, Kaek Mhong song, Patcha song, Pae song, Lao Siang Tien song, Soi Son Yhai song, Kaekmon Bangkhunpom song, Kaikeaw song, Lao Longnan song, Lao Duangduen song, Huengprai song, and Oh Duangchampa song. These songs are played during the breaks or insignificant parts of the ceremony. Towards the end of Mahesak city guardian ceremony, the musicians will manage to slowly tune out the sound of Piphat ensemble with the elegant and exquisite ending melody.
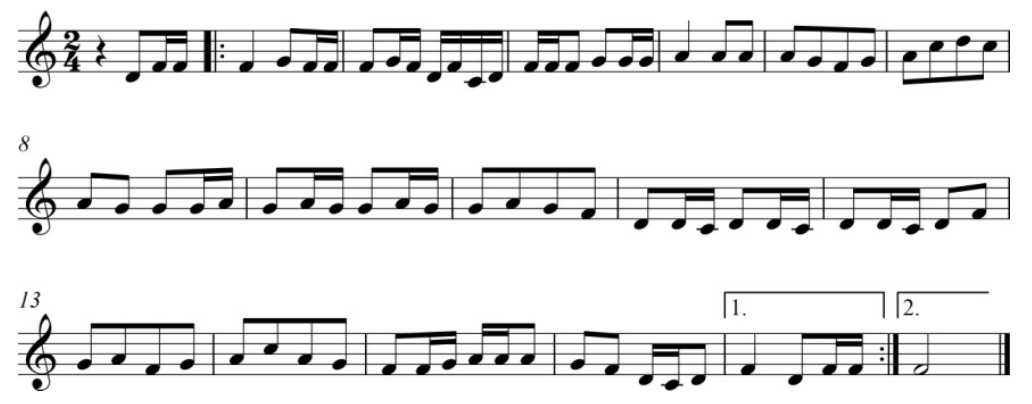

Figure 7. Melody of Patcha Song

Due to the lack of governmental supports in conservation of this precious historic ceremony, Mahesak city guardian ceremony has become less important among the new generation of Laos people. To raise the concern amongst the people of future generation, the insightful descriptive studies of various long-standing religious ceremonies should be conducted further to prevent the permanent loss of these nationally symbolistic ceremonies. 


\section{Conclusion}

Mahesak city guardian ceremony is a ritual that reflects the faith and respect of Champasak civilians toward the city guardian spirits via the exquisite sound of Piphat musical ensemble. The ceremony also involves the spiritualistic media who mediate the communication between the sacred city guardians and Champasak people. Mahesak ceremony signifies the sanctification, protection, and prosperity provided by city guardian spirits. Different songs with their unique tunes and melodies are played upon the arrival of each city guardian spirits. Therefore, Piphat ensemble is believed to serve as a medium that enables the communication between human beings and the city guardian spirits via the male and female spiritual mediators called Poe-Sang and Mae-Lam, respectively. The belief in this matter can still be found in several countries across Southeast Asia (Puntarikwiwat, 2010). Many similar factors exist amongst Southeast Asian countries, such as geographical features, climate, physical and biological environment. As a result, the strong convergent relationship in the believes among populations from the different countries appears through the similar performance of rituals and ceremonies (Groff, 1996).

\section{Acknowledgements}

I am grateful for the support and the precious research guidance from Dr. Rujee Srisombut during this research being conducted.

\section{Conflict of interests}

The authors declare that there is no conflict of interests regarding the publication of this paper.

\section{References}

Brown, M., \& Zasloff, J. J. (1976). Laos in 1975: People's Democratic Revolution--Lao Style. Asian Survey, 16(2), 193-199.

Bunpala, S. (2015). A research project and folklore performance on artistic development from spirit offerings ceremony as white-robed wearing clothes of Chow Pha. Research and Development Journal Loei Ratjabhat University, 10(33), 1-10.

Burke, A., \& Vaisutis, J. (2007). Lonely Planet Laos. Victoria, Australia: Lonely Planet Publications.

Campbell, J. (1991). The Power of Myth (p. 64). New York: Anchor.

Church, P. (Ed.). (2006). A short history of South-East Asia (Vol. XII). Singapore: John Wiley and Sons Asia.

Coedès, G. (1968). In W. F. Vella (Ed.), The Indianized States of Southeast Asia. Trans. Susan Brown Cowing.

Doungwilai, C., Hongsuwan, A. P. D. P., \& Tularak, U. (2012). The Prabang Myths: The Sacred Narratives and their Cultural Meaning1.

Evans, G. (1998). The politics of ritual and remembrance: Laos since 1975. University of Hawaii Press.

Groff, L., \& Smoker, P. (1996). Spirituality, religion, culture, and peace: Exploring the foundations for inner-outer peace in the twenty-first century. The International Journal of Peace Studies, 1(1), 57-113.

Hanna, J. L. (1988). The representation and reality of religion in dance. Journal of the American Academy of Religion, 56(2), 281-306.

Heidhues, M. F. S. (2000). Southeast Asia: A concise history. Thames and Hudson.

Pikulsri, C. (2008). Dontri Lao Derm. Khon Khaen. Center for Social Plurality, Khon Khaen University.

Puntarikwiwat, T. (2010). Buddhism and Political Society in Southeast Asia. In Kanparit S. \& Lertpaibunsiri S. (Eds.), Usakane Ti Rak (pp. 284). Bangkok.

Thammawat, C. (1985). Annals of Laos include Luang Prabang, Vientiane, Muang Puan, and Champasak (pp. 86-87). MahaSarakham: The Research Institute of Northeast Art and Culture, Srinakarinwirot University.

Wipakpotjanakit, T. (2003). History of I-san. Bangkok, Thammasat University.

Wisuttipat, M. (2014). Pipat Traditions in Music Culture in the Mekong River Basin: Practice and Phenomena in the Early 21 st Century. Fine Arts International Journal, 18(1), 12-22.

\section{Copyrights}

Copyright for this article is retained by the author(s), with first publication rights granted to the journal.

This is an open-access article distributed under the terms and conditions of the Creative Commons Attribution license (http://creativecommons.org/licenses/by/4.0/). 